\title{
Resolving the EGF-EGFR interaction characteristics through a multiple-temperature, multiple-inhibitor, real-time interaction analysis approach
}

\author{
HANNA BJÖRKELUND ${ }^{1,2}$, LARS GEDDA ${ }^{1,3}$, MAGNUS MALMQVIST ${ }^{1,2,4}$ and KARL ANDERSSON ${ }^{1,2,4}$ \\ ${ }^{1}$ Biomedical Radiation Sciences, Department of Radiology, Oncology and Radiation Sciences, \\ Rudbeck Laboratory, Uppsala University; ${ }^{2}$ Ridgeview Instruments AB, Uppsala; ${ }^{3}$ Swedish Radiation \\ Safety Authority, Stockholm; ${ }^{4}$ Ridgeview Diagnostics AB c/o Bioventia AB, Uppsala, Sweden
}

Received August 14, 2012; Accepted October 19, 2012

DOI: $10.3892 / \mathrm{mco} .2012 .37$

\begin{abstract}
Overexpression and aberrant activity of the epidermal growth factor (EGF) have been observed in various cancer types, rendering it an important target in oncology research. The interaction between EGF and its receptor (EGFR), as well as subsequent internalization, is complex and may be affected by various factors including tyrosine kinase inhibitors (TKIs). By combining real-time binding curves produced in LigandTracer ${ }^{\circledR}$ with internalization assays conducted at different temperatures and with different TKIs, the processes of ligand binding, internalization and excretion was visualized. SKOV3 cells had a slower excretion rate compared to A431 and U343 cells, and the tested TKIs (gefitinib, lapatinib, AG1478 and erlotinib) reduced the degree of internalization. The kinetic analysis of the binding curves further demonstrated TKI-dependent balances of EGFR monomer and dimer populations, where lapatinib promoted the monomeric form, while the other TKIs induced dimers. The dimer levels were found to be associated with the apparent affinity of the EGF-EGFR interaction, with EGF binding stronger to EGFR dimers compared to monomers. This study analyzed how real-time molecular interaction analysis may be utilized in combination with perturbations in order to understand the kinetics of a ligand-receptor interaction, as well as some of its associated intracellular processes. Our multiple-temperature and -inhibitor assay setup renders it possible to follow the EGFR monomer, dimer and internalized populations in a detailed manner, allowing for a new perspective of the EGFR biology.
\end{abstract}

Correspondence to: Hanna Björkelund, Biomedical Radiation Sciences, Department of Radiology, Oncology and Radiation Sciences, Rudbeck Laboratory, Uppsala University, Uppsala SE-751 85, Sweden

E-mail: hanna@ridgeview.eu

Key words: epidermal growth factor, tyrosine kinase inhibitors, internalization, kinetics, dimerization, heterogeneity

\section{Introduction}

The binding of the epidermal growth factor (EGF) to its receptor (EGFR) triggers a large set of downstream processes, ultimately causing cell growth, differentiation and proliferation $(1,2)$. The receptor interaction and subsequent events are essential for the cell, although they may also be a threat to it. The overexpression and increased activity of EGFR have been linked to a variety of tumor types (3), rendering EGFR an important target for cancer therapy. A full understanding of the biology behind the binding of EGF and its link to cancer development is thus crucial for future drug development.

Besides EGFR, the EGFR family consists of three other members: HER2, HER3 and HER4. The receptors are known to dimerize, as either homodimers or as heterodimers, with other members of the family. The extent to which the dimerization occurs and its correlation to ligand binding and further signaling have been discussed for several years, however, are yet to be fully understood (2). The binding of EGF to EGFR generates a shift in conformation, enabling EGFR to dimerize (4-6), which in turn activates the cytoplasmic tyrosine kinase domain to become activated through phosphorylation (7). Studies have also demonstrated signs of preformed EGFR dimers on the cell surface in the absence of bound EGF $(8,9)$. For example, HER2 overexpression has been associated with growth factor-independent induction of EGFR-HER2 formation (10), as well as subsequent EGFR tyrosine phosporylation $(11,12)$. HER 2 is consistently and ligand-independently activated and is the preferred binding partner of EGFR (13).

We have previously shown that the kinetic behavior of the EGF-EGFR interaction may vary greatly among cell lines (14), as demonstrated by real-time interaction measurements performed using LigandTracer ${ }^{\circledR}$ instruments. The binding curves showed at least two parallel interaction events, one fast-on-fast-off interaction and one higher affinity interaction, in which the association and dissociation rates of EGF were much slower. Thorough investigations have led to the hypothesis that the high affinity contribution of the binding curve represents EGF binding to either EGFR homodimers (EGFR-EGFR) or heterodimers (EGFR-HER2), while the weaker interaction is EGF binding to EGFR monomers (10). 
The monomeric and dimeric EGFR forms could correspond to the previously discussed high- and low-affinity EGFR receptor populations (15-17). Furthermore, there is a difference between the rates of EGF association and dissociation to EGFR homodimers and heterodimers $(10,12)$.

Tyrosine kinase inhibitors (TKI) are developed to prevent downstream signaling from EGFR. Several TKI anticancer drugs are currently available that focus on disrupting the kinase activity of EGFR $(18,19)$, including gefitinib (Iressa ${ }^{\mathrm{TM}}$ ), lapatinib (Tykerb ${ }^{\mathrm{TM}}$ ) and erlotinib (Tarceva $\left.{ }^{\mathrm{TM}}\right)$ (20-22). In theory, this causes a decrease in tumor growth, although the response varies largely between patients $(23,24)$. Certain mutations in the receptor have been shown to be predictive markers for either sensitivity or resistance $(24,25)$, although the mechanisms underlying variation between patient responses have not been completely established (26,27).

In addition to growth rate inhibition, gefitinib, erlotinib and the TKI AG1478 have been shown to promote EGFR dimerization (10,28-30). These dimers have been revealed to be non-active and conformationally different from ligand-dependent dimer forms $(10,28,30)$. By contrast, lapatinib has been shown to bind the inactive EGFR conformation and does not induce dimer formation $(31,32)$.

Previous studies have demonstrated that the presence of gefitinib affects the kinetic properties (association and dissociation rates) of the EGF-EGFR interaction in certain cell lines, observed as an increase in affinity $(10,14)$. This observation may be the result of the larger number of EGFR dimers present on the cell surface upon gefitinib treatment, regardless of their kinase activity. Lapatinib, which in contrast to gefitinib, stabilizes the inactive form of EGFR, was observed to reduce the affinity of the EGF-EGFR interaction (14).

For cells growing in physiological conditions, EGF binding and the formation of EGFR dimers induce a rapid internalization of the occupied receptors through endocytosis. Internalized ligand-receptor complexes are separated, inactivating the EGFR. Through sorting endosomes, the unphosphorylated receptors are either recycled back to the surface, or are transferred to lysosomes for degradation $(12,33,34)$. The destiny of EGFR may depend on its dimerization partner. Previous studies indicated slow or even completely disrupted EGFR internalization in case of HER2 heterodimerization (35-37), while findings of other studies $(12,37)$ claim that HER2 as a binding partner does not affect the internalization rate per se, instead the subsequent degradation processes. While EGFR homodimers are destined to a rapid lysosomal degradation, EGFR-HER2 heterodimers have been observed to be more prone to dissociate in early endosomes (37), shown as a larger fraction of recycled activated EGFRs in HER2 overexpressing cells (12).

The binding of TKIs to EGFR may also affect its internalization and degradation. In their study, Nishimura et al (27) detected a reduction of the EGFR internalization rate and a retarded transition from early to late endocytosis upon gefitinib treatment.

In interaction studies cell measurements are usually performed at either room temperature or in a cool environment, using manual end-point assays. Replacing end-point binding assays with time-resolved assays may increase the information content of the measurements, as shown in a previous study
(14). The purpose of non-physiological temperature is to slow other cell processes, such as growth, internalization, recycling and formation of new receptors, i.e., events that may influence the interaction data, thus rendering them more difficult to interpret. A drawback of restricting typical cell behavior is that the measured interaction data are obtained from artificial circumstances and may thus not always be an adequate representation of the interaction under physiological conditions.

The primary aim of this study was to investigate the molecular interactions within the EGFR system in human cancer cells, by introducing perturbations, such as temperature changes and TKIs on cell lines expressing different proportions of EGFR and HER2. A secondary goal was to develop new generic tools to investigate cell processes associated with receptor interactions, using real-time interaction measurement technology. Real-time data of the EGF-EGFR interaction from measurements conducted at cold or room temperatures, as well as in an incubator environment at $37^{\circ} \mathrm{C}$ using the human tumor cell lines A431, U343 and SKOV3 and the four TKIs gefitinib, lapatinib, AG1478 and erlotinib were demonstrated. When combining interaction data with the degree of internalization of ${ }^{125}$ I-EGF at different time points and temperature settings, we were able to hypothesize as to how EGF is bound, internalized, recycled and degraded depending on EGFR and HER2 expression, as well as treatment with TKIs.

\section{Materials and methods}

Cell culture. The human squamous carcinoma cell line A431 (CLR 1555; ATCC, Rocksville, MD, USA), the human ovarian carcinoma cell line SKOV3 (HTB-77; ATCC), the human glioma cell line U343MGaCl2:6 [a subclone of U343MG (38)], denoted U343, were used in this study. Cells were selected to represent a range of EGFR and HER2 expression: 2E6 EGFR/ cell and 2E5 HER2/cell for A431, 6E5 EGFR/cell and 3E4 HER2/cell for U343 and 3E5 EGFR/cell and 2E7 HER2/ cell for SKOV3 (10). HER3 and HER4 populations are considered small enough on the cell surface to be neglected $(30,39)$. EGF has previously been confirmed to bind specifically to EGFR using different assays (40-42).

The cells were cultured in Ham's F10 (A431 and U343) or RPMI (SKOV3) cell culture medium (Biochrom AG, Berlin, Germany), supplemented with $10 \%$ fetal calf serum (FCS) (Sigma-Aldrich, St. Louis, MO, USA), L-glutamine (2 mM) and PEST (penicillin $100 \mathrm{IU} / \mathrm{ml}$ and streptomycin $100 \mu \mathrm{g} / \mathrm{ml}$, Biochrom $\mathrm{AG}$ ) in a humified incubator at $37^{\circ} \mathrm{C}$, equilibrated with $5 \% \mathrm{CO}_{2}$. The cells were seeded on a local area of a cell culture dish (Nunclon ${ }^{\mathrm{TM}}$, size 100x20; NUNC A/S, Roskilde, Denmark) for the LigandTracer measurements, as described previously (43).

Environments. The measurements described below were conducted i) at room temperature, $\sim 22^{\circ} \mathrm{C}$ with normal $\mathrm{CO}_{2}$ levels; ii) in a humified incubator, at $37^{\circ} \mathrm{C}$ with $5 \% \mathrm{CO}_{2}$; or iii) in a cold room, at $\sim 7^{\circ} \mathrm{C}$.

Radiolabeling. Human EGF (Chemicon International, Temecula, CA, USA) was labeled with ${ }^{125}$ I (Perkin-Elmer, Wellesley, MA, USA), using the chloramine-T protocol (44). 
Chloramine-T (Sigma-Aldrich, St. Louis, MO, USA) and sodium metabisulfite (Sigma-Aldrich, Stockholm, Sweden) were used for the labeling reactions. Excess of ${ }^{125} \mathrm{I}$ and reagents were separated from the labeled EGF solution using a NAP-5 column (GE Healthcare, Waukesha, WI, USA) equilibrated with phosphate-buffered saline (PBS) $(10 \mathrm{mM}, \mathrm{pH} 7.4$, $140 \mathrm{mM} \mathrm{NaCl})$.

Treatment of cells with TKIs. Cells were treated for $48 \mathrm{~h}$ in $1 \mu \mathrm{M}$ of either gefitinib (Biaffin $\mathrm{GmbH} \&$ Co KG, Kassel, Germany), lapatinib, AG1478 or erlotinib (all three from LC Laboratories, Woburn, MA, USA) prior to binding studies in LigandTracer, acid wash measurements or cell counting. After the first $24 \mathrm{~h}$ of treatment, fresh medium containing TKI was added to ensure a continuous supply.

Real-time measurements of the ${ }^{125} I$-EGF-EGFR interaction at room temperature and in an incubator. The ${ }^{125}$ I-EGF-EGFR interaction was detected in real-time on cultured tumor cells, using two LigandTracer Grey instruments in parallel, one of which was placed at room temperature, and the other in an incubator at $37^{\circ} \mathrm{C}$. The incubator instrument was temperature-equilibrated $1 \mathrm{~h}$ prior to measurement. The technology behind and the validation of LigandTracer has been described previously (43,45). The uptake of 2.7 (A431), 1.5 (U343) and 2 $\mathrm{nM}$ (SKOV3) was measured for either 2 or $4 \mathrm{~h}$. The concentrations were sufficient to ensure $<10 \%$ loss of free ${ }^{125}$ I-EGF in solution due to binding, reducing the risk of depletion affecting the results. The shorter uptake phase $(2 \mathrm{~h})$ was followed by a $2 \mathrm{~h}$ retention measurement. This procedure was performed using either normally treated cells grown in complete culture medium or cells pre-treated with TKI. In the latter case, the same TKI was included in the medium during the measurement. Note that the term 'uptake' refers to the sum of the events occurring during incubation, i.e., when ${ }^{125}$ I-EGF is present, while 'retention' is the combination of events taking place once the ${ }^{125}$ I-EGF solution has been removed and replaced with fresh medium. Examples of biological events that affect measured ${ }^{125} \mathrm{I}$ signal are EGF association and dissociation to/ from EGFR and the excretion of ${ }^{125}$ ( $\left({ }^{125}{ }^{12}\right.$ I-EGF) from the cell, once the ligand has been internalized.

Each measurement was repeated at least twice, essentially under the same experimental conditions. Obtained curves were evaluated using TraceDrawer 1.4 (Ridgeview Instruments AB, Uppsala, Sweden).

Acid wash measurements used to estimate membrane bound and internalized ${ }^{125} I-E G F$. Cultured A431, U343 and SKOV3 cells were seeded in 12-well plates (Nunclon, size 100x20; NUNC A/S). Forty-eight hours prior to measurements, the cells were grown in a normal, TKI-free environment or in complete cell culture medium containing $1 \mu \mathrm{M}$ of gefitinib, lapatinib, AG1478 or erlotininb. Cells were incubated with ${ }^{125}$ I-EGF for 40, 90 or $240 \mathrm{~min}$ at either room temperature or $37^{\circ} \mathrm{C}$, using the same concentrations as in the LigandTracer incubator measurements. After a washing step, the cells were treated with acid in $0.2 \mathrm{M} \mathrm{Gly-HCl} \mathrm{pH} 2.5$ containing $0.15 \mathrm{M} \mathrm{NaCl}$ and $4 \mathrm{M}$ Urea for $5 \mathrm{~min}$ on ice to strip the cell surface-associated ${ }^{125}$ I-EGF from the membrane. Then, $1 \mathrm{M}$ $\mathrm{NaOH}$ was added and incubated at $37^{\circ} \mathrm{C}$ for at least $1 \mathrm{~h}$ to fully disrupt the structure of the cells, thus enabling collection of internalized ${ }^{125} \mathrm{I}$-EGF. The activity from the solutions was measured using an automatic gamma counter (1480 WIZARD $^{\text {TM }}$ 3", PerkinElmer, Waltham, MA, USA).

To obtain further information concerning the EGF-EGFR interaction, a few combinations of cells and treatments were studied after $90 \mathrm{~min}$ incubation on ice or at $7^{\circ} \mathrm{C}$ in a cold room.

Effects of TKIs on the kinetics of the ${ }^{125}$ I-EGF-EGF interaction at room temperature and at $7^{\circ} \mathrm{C}$. A stepwise increase of ${ }^{125} \mathrm{I}$-EGF concentration was monitored at room temperature or inside a cold room $\left(7^{\circ} \mathrm{C}\right)$ using LigandTracer Grey, as described previously (14). Instruments and liquids were temperature equilibrated before measurements were conducted in the cold room. The set of studied concentrations were cell type-specific and selected to match the previously estimated affinity of the EGF-EGFR interaction at room temperature in the three hosting cell lines (2.7,9 and $27 \mathrm{nM}$ for A431; 0.5, 1.5 and $4.5 \mathrm{nM}$ for U343 and 0.7, 2 and $6 \mathrm{nM}$ for SKOV3) (14). As cell detachment may be a problem for cells exposed to low temperatures for a longer time period, only the two highest concentrations were used in cold room measurements to shorten the uptake phase. The binding of ${ }^{125}$ I-EGF was followed for $2.5 \mathrm{~h}$ for each concentration, followed by a retention measurement overnight using fresh medium. Cell detachment in cold room was estimated after $5 \mathrm{~h}$ of retention measurement by counting cells in the media. TKI pre-treated cells had the same inhibitor present in the medium throughout the run.

Interaction map analysis. Data produced in LigandTracer were analyzed using the Interaction Map $^{\circledR}$ (IM) method (Ridgeview Diagnostics AB, Uppsala, Sweden) to investigate the heterogeneity of the EGF-EGFR interaction, as described previously $(10,46,47)$. IM deciphers the different components of a measured interaction from the shape of its real-time binding curve. Each component is a contributing interaction describing one type of ligand binding to one type of target. The components are presented as areas in an on-off map (48) with darkness relative to their weighing factors, i.e., degree of contribution to the measured binding curve.

TKI effects in cell count. Tumor cells were cultivated in 12-well plates and treated with TKIs for $48 \mathrm{~h}$ prior to the addition of a trypsin-EDTA solution (Biochrom AG) at $37^{\circ} \mathrm{C}$ to cause cell detachment. Cells were counted to estimate growth-disturbing effects caused by TKIs in A431, U343 and SKOV3 cells.

\section{Results}

Real-time measurement data of the ${ }^{125} I-E G F-E G F R$ interaction in incubator shows signs of internalization and processing of ligand. Data from real-time measurements of the binding of ${ }^{125}$ I-EGF are shown in Fig. 1. The interaction between ${ }^{125}$ I-EGF and A431 (Fig. 1A and B), U343 (Fig. 1C and D) or SKOV3 (Fig. 1E and F) cells was followed at room temperature (black curves) or inside an incubator at $37^{\circ} \mathrm{C}$ (grey curves), for $4 \mathrm{~h}$ (solid line) or $2 \mathrm{~h}$, followed by a $2 \mathrm{~h}$ retention measurement (dotted line; mark indicates the start of this retention phase). Curves have been normalized at $\mathrm{t}=10 \mathrm{~min}$ to minimize the impact on signal level due to variations in the receptor number 
A

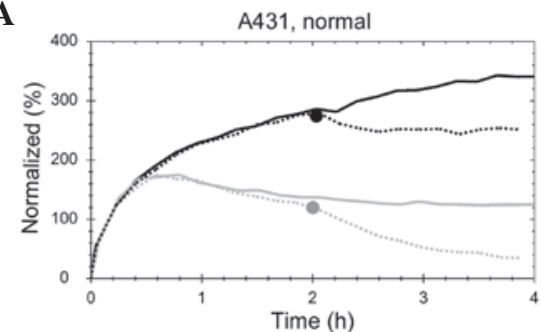

C

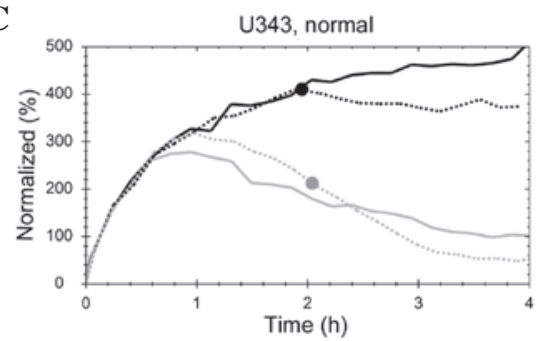

$\mathbf{E}$

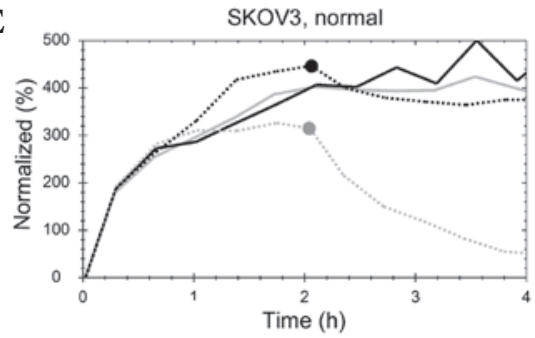

B

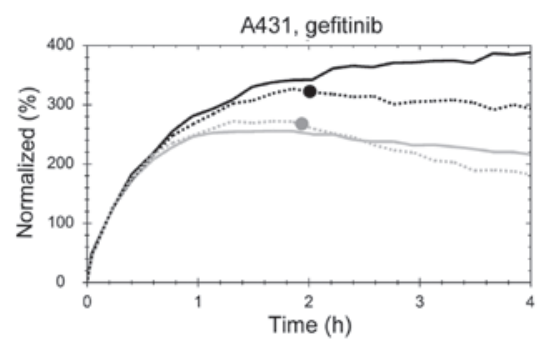

D

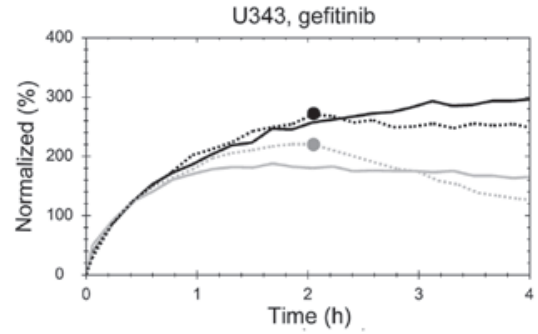

F

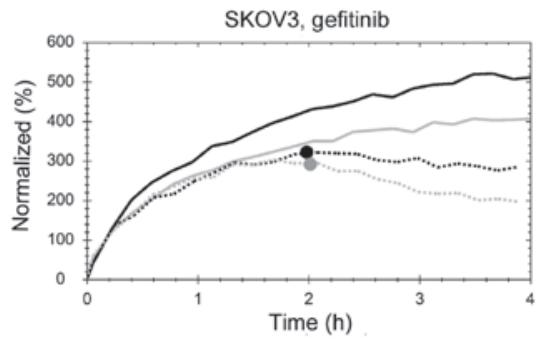

Figure 1. Interaction between ${ }^{125} \mathrm{I}$-EGF and (A and B) A431, (C and D) U343 or (E and F) SKOV3 cells at room temperature (black curves) or inside an incubator at $37^{\circ} \mathrm{C}$ (grey curves). Cells were incubated for either 4 (solid line) or, $2 \mathrm{~h}$, followed by a retention measurement in which the ligand solution was exchanged with pure medium (dotted line; mark indicates the start of this retention phase). Measurements were performed with control cells grown under normal conditions (A, C and E) or in $1 \mu \mathrm{M}$ of either gefitinib, lapatinib, AG1478 or erlotinib, here represented by gefitinib (B, D and F) as the effects were similar for all tyrosine kinase inhibitors (TKIs).

caused by differences in cell counts. The measurements were performed under either normal conditions, with control cells grown in complete cell culture medium, or in the presence of $1 \mu \mathrm{M}$ of gefitinib, lapatinib, AG1478 or erlotinib. The effects of TKI on the cell uptake of ${ }^{125} \mathrm{I}-\mathrm{EGF}$ at $37^{\circ} \mathrm{C}$ compared to room temperature were similar between the four TKIs and are represented by data from the gefitinib effect measurements (Fig. 1B, D and F).

When ${ }^{125}$ I-EGF was added to A431 or U343 cells at $37^{\circ} \mathrm{C}$, the signal initially increased rapidly due to binding of ${ }^{125} \mathrm{I}-\mathrm{EGF}$ to membrane bound EGFR. After $\sim 40 \mathrm{~min}$ the increase was reduced and the signal started to decrease as EGFR with bound ${ }^{125}$ I-EGF likely was internalized and degraded, inducing an excretion of the nuclide. In SKOV3 cells no clear decrease was visible in signal, suggesting that some (or several) of the cell processes that evenutally led to excretion of ${ }^{125} \mathrm{I}$ were considerably slower in the SKOV3 compared to the A431 or U343 cells.

Temperature changes may affect the kinetics of an interaction. This was clearly observed for the dissociation of ${ }^{125} \mathrm{I}$ from SKOV3 cells, which accelerated with increased temperature.

When treated with gefitinib, lapatinib, AG1478 or erlotinib, the differences between room temperature and incubator measurements were reduced. The clear decrease in signal observed in TKI untreated A431 and U343 cells was small or non-existent in the presence of any of the four TKIs (Fig. 1B and D). This finding indicates that either the internalization rate or intracellular degradation was affected by the TKIs.
In SKOV3 cells, the effect of TKIs (Fig. 1F) was not as clear as in A431 or U343 cells. The main difference was observed in the dissociation rate, which was slower when cells had been treated with lapatinib (data not shown) and notably slower with gefitinib, AG1478 and erlotinib.

Acid wash measurements demonstrated a decrease in the amount of internalized ${ }^{125}$ I-EGF in A431, U343 and SKOV3 cells induced by TKIs. Data from acid-and subsequently base-treated cells show the amount of membrane-bound and internalized ${ }^{125} \mathrm{I}$-EGF, respectively. The average fraction of the internalized activity of total cell-associated activity (membrane bound + internalized) at room temperature or at $37^{\circ} \mathrm{C}$ (inside an incubator) at different time points is shown in Fig. 2. Error bars show the distribution of the triplicates, (i.e., maximum value-minimum value)/2. For the three cell lines, the TKIs significantly decreased the fraction of internalized ${ }^{125} \mathrm{I}$ activity at both room temperature and at $37^{\circ} \mathrm{C}$, although to a lesser extent by erlotinib. AG1478 in A431 and SKOV3 cells, and lapatinib in U343 cells, also showed a lower impact on the internalization degree.

The total cell-associated activity was plotted over time (data not shown) and matched the real-time binding curves from LigandTracer measurements at room temperature and $37^{\circ} \mathrm{C}$ well (Fig. 1), which serves as a quality control for the two methods. The degree of internalization was similar at room temperature and at $37^{\circ} \mathrm{C}$, for the three cell lines, or even higher at room temperature. 
A

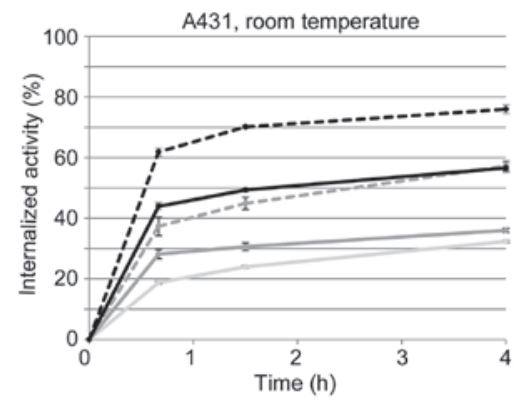

C

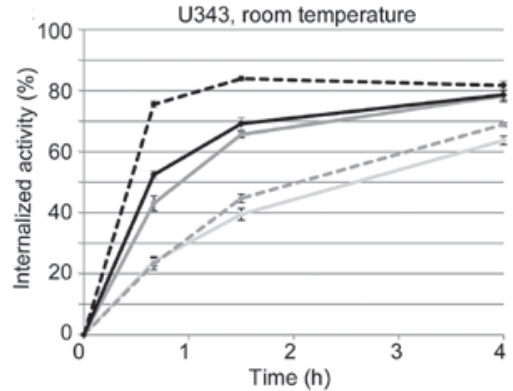

E

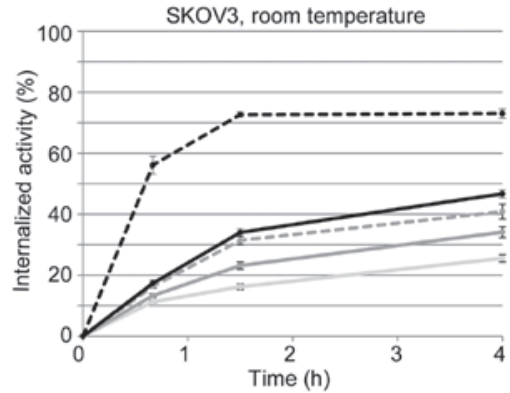

B

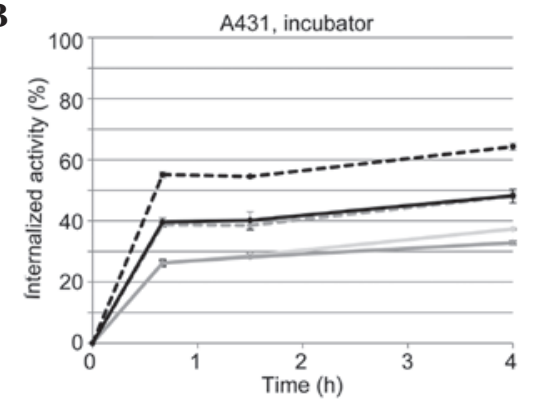

D

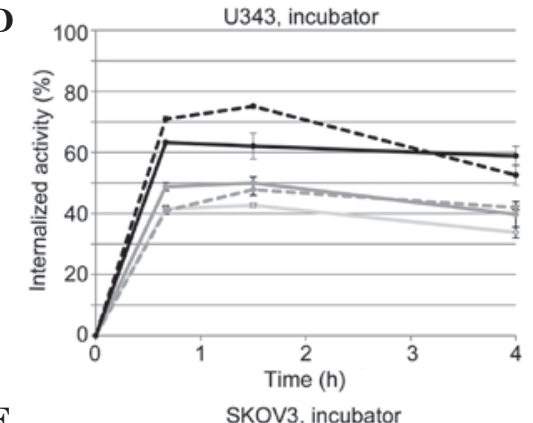

F

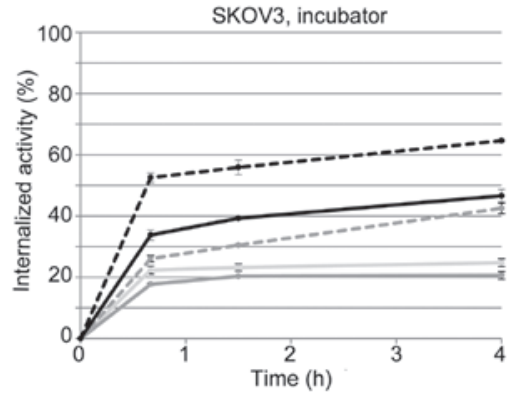

Figure 2. Internalized activity of ${ }^{125}$ I-EGF as percentage of total cell-associated activity for cultured (A and B) A431, (C and D) U343 and (E and F) SKOV3 cells, grown either in normal cell culture medium (black, dashed line) or pre-treated with $1 \mu \mathrm{M}$ of gefitinib (light grey, solid line), lapatinib (grey, solid line), AG1478 (grey, dashed line) or erlotinib (black, solid line). The tyrosine kinase inhibitors (TKIs) reduced the degree of internalized activity, most notably observed for gefitinib, lapatinib and AG1478. Error bars show the distribution of triplicates, (maximum value-minimum value)/2.

Table I. Degree of internalization at $7^{\circ} \mathrm{C}$ and on ice.

\begin{tabular}{llc}
\hline Cell line & $7^{\circ} \mathrm{C}(\%)$ & On ice $(\%)$ \\
\hline A431 & $6.0 \pm 0.3$ & $2.2 \pm 0.3$ \\
A431+gef & $5.1 \pm 0.4$ & $2.8 \pm 0.1$ \\
U343 & $4.5 \pm 0.2$ & $1.7 \pm 0.3$ \\
SKOV3 & $4.2 \pm 0.1$ & $1.5 \pm 0.1$ \\
\hline
\end{tabular}

The percentage of total cell-associated activity present inside the cells was measured after incubation with ${ }^{125}$ I-EGF for $90 \mathrm{~min}$ in a cold room $\left(7^{\circ} \mathrm{C}\right)$ or on ice. Errors are presented as the distribution of triplicates, i.e., (maximum value-minimum value)/2. Gef, gefitinib.

A relatively large amount of ${ }^{125} \mathrm{I}$-EGF was internalized in SKOV3 cells (Fig. 2E-F) at both temperatures $(\sim 70 \%$ of total cell-associated activity), similar to what was observed in A431 cells (Fig. 2A and B).

The fraction of internalized ${ }^{125}$ I-EGF of the cell associated ${ }^{125} \mathrm{I}$-EGF was constant or increased slightly over time for A431 and SKOV3 cells at $37^{\circ} \mathrm{C}$, for the treatments (Fig. 2B and F). For U343 cells the degree of internalized activity was constant or decreased (Fig. 2D). The decrease indicates that the degradation and excretion of ${ }^{125}$ I-EGF was faster than the internalization.

When incubated inside a cold room $\left(7^{\circ} \mathrm{C}\right)$ or on ice, the percentage of cell-associated ${ }^{125}$ I-EGF inside the cell was $\sim 5$ and $2 \%$, respectively (Table I).

TKIs affect the kinetics of the ${ }^{125} I-E G F-E G F R$ interaction albeit differently, depending on the inhibitor and cell line. The uptake and retention of ${ }^{125}$ I-EGF were detected using a stepwise increase of the ${ }^{125} \mathrm{I}$-EGF concentration, three concentrations at room temperature and two for the measurements performed at $7^{\circ} \mathrm{C}$ in a cold room (Fig. 3). The curves were normalized at the beginning of the final incubation to remove any signal magnitude differences originating from total receptor number related to cell count. When comparing the binding of ${ }^{125}$ I-EGF to the differently treated cells, the shape of the curve at each uptake and retention phase is important, as well as the manner in which the signal increases at each addition of ${ }^{125}$ I-EGF.

Gefitinib (light grey, solid line), AG1478 (grey, dotted line) and erlotinib (black, solid line) clearly reduced the dissociation rate, or possibly disrupted the fast-off component of the interaction in $\mathrm{A} 431$ cells at both room temperature and $7^{\circ} \mathrm{C}$ 


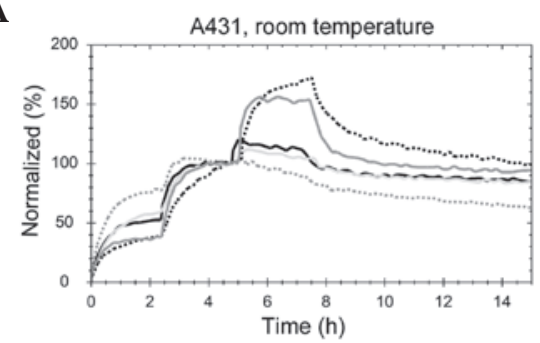

C

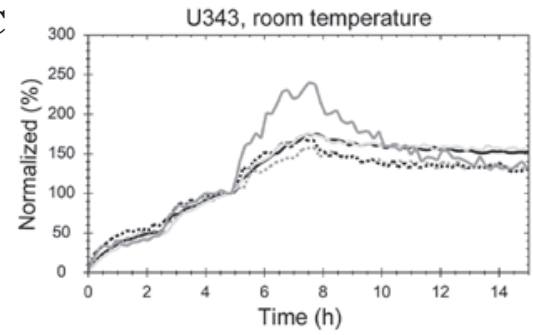

$\mathbf{E}$

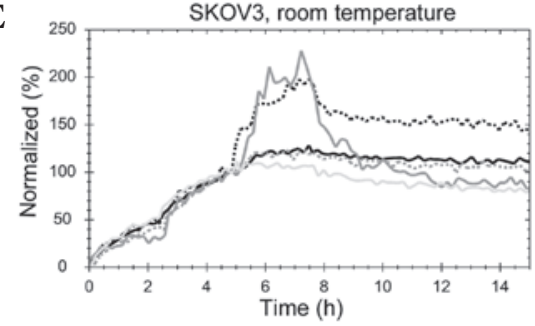

B

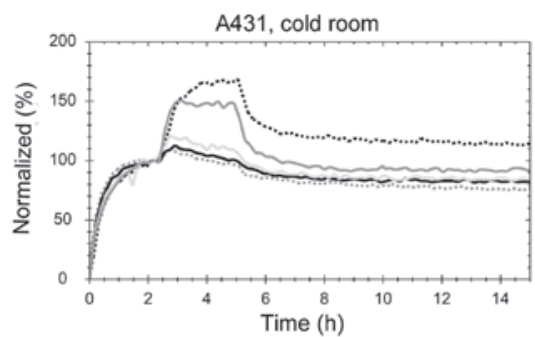

D

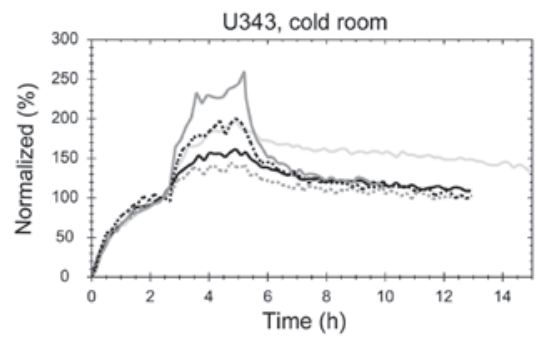

F

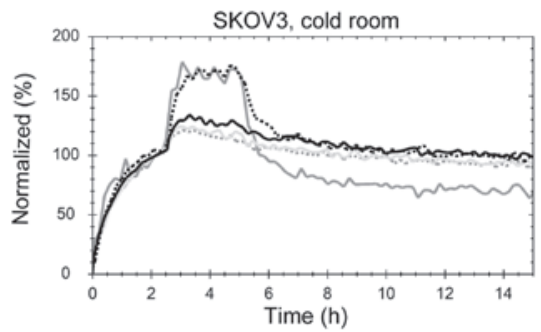

Figure 3. Real-time binding curves of the ${ }^{125}$ I-EGF-EGFR interaction measured in (A) A431 at room temperature, (B) A431 at $7^{\circ} \mathrm{C}$, (C) U343 at room temperature, (D) $\mathrm{U} 343$ at $7{ }^{\circ} \mathrm{C}$, (E) SKOV3 at room temperature or (F) SKOV3 cells at $7^{\circ} \mathrm{C}$. ${ }^{125} \mathrm{I}$-EGF was detected using stepwise increase to match previously estimated overall affinity: 2.7, 9 and $27 \mathrm{nM}$ for A431 cells ( 9 and $27 \mathrm{nM}$ at $\left.7^{\circ} \mathrm{C}\right) ; 0.5,1.5$ and $4.5 \mathrm{nM}$ for $\mathrm{U} 343 \mathrm{cells}\left(1.5\right.$ and $4.5 \mathrm{nM}$ at $\left.7{ }^{\circ} \mathrm{C}\right)$ and $0.7,2$ and $6 \mathrm{nM}$ for SKOV3 cells ( 2 and $6 \mathrm{nM}$ at $7^{\circ} \mathrm{C}$ ). The cells were grown either in normal cell culture medium (black, dotted line) or pre-treated with $1 \mu \mathrm{M}$ of gefitinib (light grey, solid line), lapatinib (grey, solid line), AG1478 (grey, dotted line) or erlotinib (black, solid line).

(Fig. 3A and B). Furthermore, the receptors were almost fully saturated already after the second concentration $(9 \mathrm{nM})$, as low or no increase was observed in formed complexes for the third concentration. This finding indicates that $9 \mathrm{nM}$ is considerably higher compared to the apparent $\mathrm{K}_{\mathrm{D}}$ of the ${ }^{125}$ I-EGF-EGFR interaction, when A431 cells are treated with gefitinib, AG1478 or erlotinib. AG1478 increased the affinity the most, observed as a low increase in signal when adding the second concentration at room temperature. This finding indicates that as little as $2.7 \mathrm{nM}$ of ${ }^{125} \mathrm{I}-\mathrm{EGF}$ is enough to make most of the receptors have an EGF bound, when A431 cells are treated with AG1478. Lapatinib modified the interaction in a completely different manner. The increases in signal following the addition of ${ }^{125} \mathrm{I}-\mathrm{EGF}$ were similar to the control cells, although the time to reach equilibrium was considerably shorter. The TKI made the faster contributing interaction even faster, and increased the stability of the slower interaction (resulting in a slower dissociation rate).

In U343 cells, small effects of gefitinib, AG1478 and erlotinib on the ${ }^{125} \mathrm{I}$-EGF-EGFR interaction were visible at room temperature (Fig. 3C). Lapatinib caused a faster dissociation of ${ }^{125} \mathrm{I}-\mathrm{EGF}$ and amplified the degree of signal increase at each concentration, both of which indicate a lower affinity in the interaction between ligand and receptor. At $7^{\circ} \mathrm{C}$, gefitinib, AG1478 and erlotinib increased the affinity of the ${ }^{125}$ I-EGF-EGFR interaction, observed as a slower dissociation and (for AG1478 and erlotinib) a larger fraction of EGFR bound by low EGF concentrations (Fig. 3D).
In SKOV3 cells treated with gefitinib, AG1478 and erlotinib, the ${ }^{125}$ I-EGF-EGFR interaction had a higher affinity compared to control cells at both room temperature and $7^{\circ} \mathrm{C}$, most clearly visible as a lower increase in signal at each addition of ${ }^{125} \mathrm{I}$-EGF. Lapatinib treatment made the fast-on-fast-off contribution of the binding of ${ }^{125} \mathrm{I}$-EGF more abundant, shifting the overall interaction into a lower affinity state (Fig. 3E and F).

The fact that the binding curves obtained at room temperature and at $7^{\circ} \mathrm{C}$ are similar and the fact that ${ }^{125} \mathrm{I}-\mathrm{EGF}$ is internalized to a larger degree at room temperature compared to $7^{\circ} \mathrm{C}$ suggests that there is a recycling of ${ }^{125} \mathrm{I}$-EGF-EGFR complexes and that this process is more rapid than the dissociation rate of the ${ }^{125} \mathrm{I}$-EGF-EGFR complexes.

In general, TKIs are capable of modifying the interaction between EGF and its receptor. Gefitinib, AG1478 and erlotinib sometimes increased the affinity of the interaction, although the magnitiude of the affinity increase varied among the host cell lines. Lapatinib behaved in a markedly different way, decreasing the overall affinity and sometimes increasing the rate of ${ }^{125} \mathrm{I}-\mathrm{EGF}$ association and dissociation.

Real-time interaction data produced at room temperature were further analyzed using IM (Fig. 4). This method analyzes the shape of a binding curve and deciphers the mixture of contributing interactions possibly resulting in such a curve shape. Each interaction is presented as an area in an on-off map, where the coordinate represents the kinetic properties $\mathrm{k}_{\mathrm{a}}$ and $\mathrm{k}_{\mathrm{d}}$, and the darkness the extent to which the interaction contributes to the measured real-time binding curve. The 
A

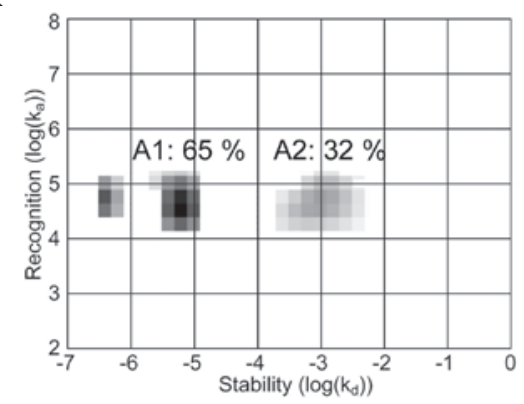

B

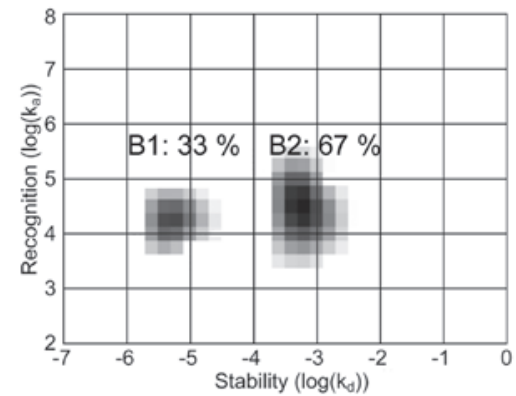

C

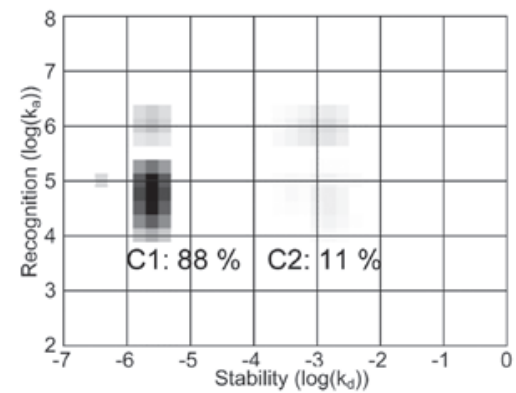

Figure 4. Interaction Maps (IM) describing the heterogeneous binding of EGF to (A) normally-, (B) lapatinib- or (C) erlotinib-treated SKOV3 cells. Maps were calculated from real-time binding data produced in LigandTracer at room temperature. The data show two present interactions with approximately the same recognition/association rate $\left(\mathrm{k}_{\mathrm{a}}\right)$, but with different stability/ dissociation rates. The contribution of the lesser stable interaction (B2) increased when SKOV3 cells were treated with lapatinib. Gefitinib, AG1478 and erlotinib (represented by erlotinib) shifted the interaction to the more stable state $(\mathrm{C} 1)$.

effects of gefitinib, AG1478 and erlotinib on the EGF-EGFR interaction were almost identical and are represented by the erlotinib treatment. Similarly, TKI effects were comparable in the three cell lines and depicted by the binding of EGF to SKOV3 cells. The contributions of the interactions were calculated by integration of the respective areas in the map.

The EGF-EGFR interaction measured in A431, U343 and SKOV 3 cells was heterogeneous, observed as two major areas in the maps. The areas, corresponding to parallel EGF-EGFR interactions, represent various dissociation rate constants of $k_{d}$. The contribution of the lesser stable interaction to the measured curve increased from 32 to $67 \%$ when SKOV3 cells were treated with lapatinib (B2). The presence of gefitinib, AG1478 and erlotinib induced the reverse effect, making the higher-affinity interaction (C1) more common.

Gefitinib, lapatinib, AG1478 and erlotininb decrease cell growth differently depending on cell line. Cells grown in 12-well plates were treated with gefitinib, lapatinib, AG1478 or erlotininb for $48 \mathrm{~h}$. The number of cells were counted in

Table II. Effect of gefitinib, lapatinib, AG1478 and erlotinib on cell growth.

\begin{tabular}{lccc}
\hline & \multicolumn{3}{c}{ Cell number } \\
\cline { 2 - 4 } & A431 (\%) & U343 (\%) & SKOV3 (\%) \\
\hline Gefitinib & $52.7 \pm 7.2$ & $70.4 \pm 6.4$ & $67.7 \pm 12.9$ \\
Lapatinib & $36.8 \pm 3.1$ & $27.0 \pm 4.2$ & $67.7 \pm 6.9$ \\
AG1478 & $30.2 \pm 3.3$ & $28.2 \pm 4.8$ & $63.6 \pm 3.9$ \\
Erlotinib & $23.3 \pm 4.3$ & $66.6 \pm 13.1$ & $55.5 \pm 5.6$ \\
\hline
\end{tabular}

Cell numbers after $48 \mathrm{~h}$ treatment of tyrosine kinase inhibitors, presented as ratios using normally treated cells as a standard (corresponding to $100 \%$ ). Errors calculated as (maximum value-minimum value) $/ 2$ of the cell count, with quadratic propagation of error.

triplicates and compared with control cells grown in complete medium, for a rough estimation of growth effects caused by the TKIs. Table II shows the average cell count for each treatment, in relation to cell count for the control (set to 100\%). Errors are presented as (maximum value-minimum value)/2 of the cell count, with quadratic propagation of error. In this experiment, the four TKIs decreased cell growth to various extents, depending on the inhibitor and cell line. SKOV3 shows a similar sensitivity to the four TKIs, reducing the growth rate to in average 55-68\%. In A431 and U343, however, the effect varied markedly, with some extreme cases where the cell count was only $25-30 \%$ of the control.

\section{Discussion}

The aim of this study was to further investigate the interaction between EGF and its receptor and to use interaction analysis at different temperatures as a new method for resolving parts of the receptor biology. Although a temperature shift towards $37^{\circ} \mathrm{C}$ renders interaction data on cells markedly more complicated, this was deemed an important development for a proper understanding of the processes involving the EGFR system. The data described in this study are complex and demonstrate several underlying processes: the association and dissociation rates of the EGF-EGFR interaction itself, as well as related events, such as dimerization, internalization, recycling and degradation of the receptor and its ligand. Thus, this paper describes a first attempt to separate the velocities of these processes, at normal conditions and in the presence of TKIs to identify the rate-limiting steps of EGFR-related biology.

The detection technology used in this study relies on quantification of a label, in this case ${ }^{125} \mathrm{I}$. Thus, the measurements follow the path of the label only. Several of the mechanistic steps in the EGF-EGFR interaction are conducted in a manner that cannot easily be resolved. The interaction analysis method used will not distinguish between surface-bound and internalized ligands, and the only way to separate the signal from such events is through perturbation with agents and conditions (in the present case TKIs and temperature) known to impact the processes in different ways, as well as cell lines with different proportions of the receptor components. Furthermore, when several events are mixed into one measurement step, the 


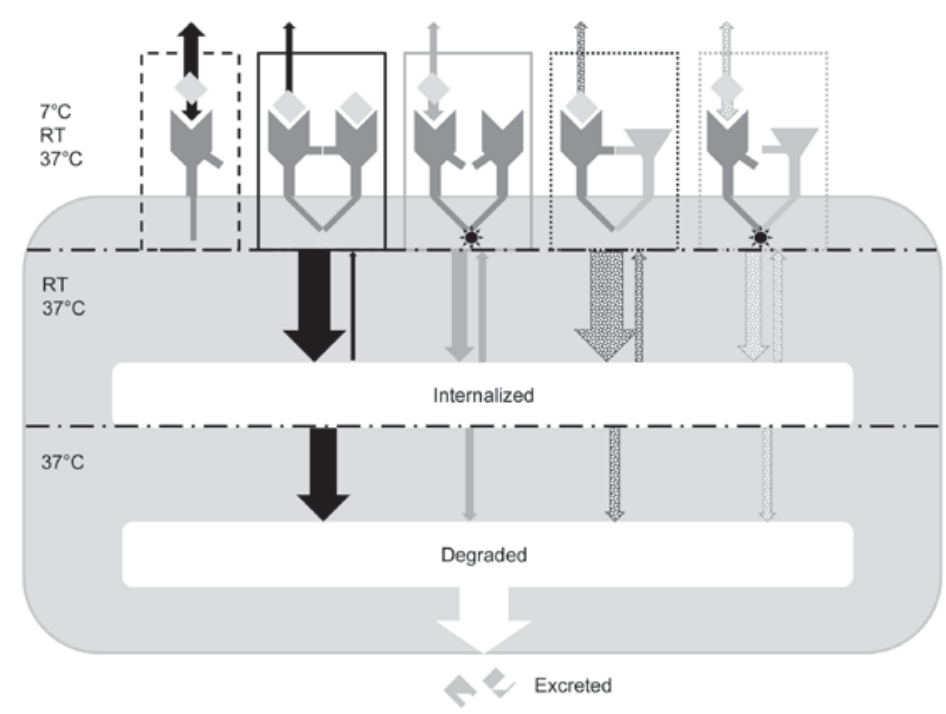

Figure 5. Proposed mechanism of EGF-GFR kinetics, internalization, recycling, degradation and excretion with regard to temperature and tyrosine kinase inhibitors (TKIs) treatment. EGFR monomers (black square, dashed line) mainly forms homodimers (black square, solid line) on A431 and U343 cells and EGFR-HER2 heterodimers (black square, dotted line) on SKOV3 cells upon EGF binding, where the choice of dimer partner depends on overall EGFR/HER2 expression. Gefitinib, AG1478 and erlotinib induce dimers (grey squares, solid and dotted lines) that are catalytically inactive. EGF dissociates slower from EGFR dimers compared to monomers. Internalization occurs at room temperature and $37^{\circ} \mathrm{C}$, but not at $7^{\circ} \mathrm{C}$. EGFR homodimers are degraded and EGF residues and ${ }^{125} \mathrm{I} \mathrm{excreted} \mathrm{at} 37^{\circ} \mathrm{C}$, but not at room temperature (RT). No or little excretion was observed on SKOV3 cells, indicating that EGFR-HER2 heterodimers are degraded to a lesser extent. The interaction between gefitinib, AG1478 or erlotinib and EGFR perturbs internalization and possibly also degradation. Lapatinib (not shown) stabilizes the monomeric EGFR form, which may explain the lower amount of internalized EGF as internalization is likely restricted to EGFR dimers. EGFR recycling is probable, but has not been investigated in detail.

rate-limiting process dictates the detected output. Thus, the measurements presented in this study constitute a complex pattern of complementing experiments, aiming at deconvoluting the underlying processes.

For A431 and U343 cells grown in the absence of TKIs, the signal from ${ }^{125} \mathrm{I}$-EGF associated with cells started to decrease after $\sim 40 \mathrm{~min}$. Consequently, this indicated ${ }^{125}$ I-EGF to be associated with EGFR, further receptor dimerization, internalization and at a later stage the degradation and excretion of EGF and its nuclide. The combined results from LigandTracer and acid-wash measurements at room temperature and $37^{\circ} \mathrm{C}$ indicated that the main difference between the two temperatures (in the absence of TKIs) was not the internalization itself, but the rate of subsequent degradation and excretion of ${ }^{125}$ I-EGF once inside the cells. These degradation processes become much slower when going from $37^{\circ} \mathrm{C}$ to room temperature. For HER2 overexpressing SKOV3 cells, the internalization mechanisms were active, although the degradation processes were slow or even disrupted at $37^{\circ} \mathrm{C}$. This clarifies which of the cell processes are affected when HER2 serves as a dimer partner, as discussed previously $(12,35,36)$.

When comparing cold conditions to room temperature the interaction data is similar, however, the internalization is turned off at $7^{\circ} \mathrm{C}$. We thus conclude that the internalization and recycling processes are more rapid compared to the dissociation rate of the EGF-EGFR complex, rendering internalization and recycling difficult to detect by interaction analysis alone at room temperature.

When cells were treated with any of the four TKIs gefitinib, lapatinib, AG1478 or erlotinib, the LigandTracerbinding curves from the incubator measurements became more similar to data from the room temperature measurements, indicating that the degree of either nuclide excretion or internalization was reduced (Fig. 1). Acid wash measurements provided additional information by showing that the degree of internalization was significantly lower in the three cell lines in the presence of TKIs, although most likely not low enough to cause the similarity of $37^{\circ} \mathrm{C}$ to room temperature. Thus, the TKIs are believed to shift the balance towards receptor complexes on the cell surface, as well as to reduce the speed of the excretion.

Measurements of the impact of TKIs on interaction kinetics revealed that gefitinib, AG1478 and erlotinib increased the affinity in A431 and SKOV3 cells, while lapatinib decreased it in the three cell lines. Gefitinib, AG1478 and erlotinib have been shown to cause growth factor-independent formation of EGFR dimers, while lapatinib strengthens the inactive monomeric form of EGFR (10,28-32). The correlation between affinity increases and dimerization induction strengthens previous hypothesis that EGF binds to dimeric EGFR forms with a higher affinity compared to the EGFR monomer $(10,17)$. Notably, this is not associated with internalization and degradation perturbations, as all four TKIs had an effect on these events (Fig. 1B and D and 2).

Translating real-time binding data into IMs demonstrated the heterogeneity of the EGF-EGFR interaction further. Through experiments with dimerization-preventing agents, the authors previously hypothesized that the two main areas in the maps correspond to EGF interacting with either EGFR monomers or dimers, where the dimer interaction was assumed to be the more stable one (Fig. 4; A1, B1 and C1, ) (10). TKI effects investigated in this study confirm the identification of these two map areas. The IM method provides means to quantify 
the effects on dimerization caused by TKIs. The quantification is fast and straightforward and may be considered to be a convenient alternative to more time-consuming manual dimerization determining methods, such as immunoblotting. Additionally, this quantification is based entirely on the function of the receptor forms (i.e., binding abilities) and not their structures per se, in contrast to several manual alternatives.

From the findings described above, we formed a hypothesis on the pattern of EGF-binding kinetics, internalization, recycling, degradation and excretion in the presence of TKIs at different temperatures (Fig. 5). EGF may interact with EGFR monomers present on the cell surface (black, dashed line), enabling EGFR to dimerize, likely as homodimers on EGFR-rich A431 and U343 cells (black, solid line) and HER2 heterodimers on SKOV3 cells, which have a high HER2 expression (black, dotted line). EGF was suggested to possibly associate to as well as dissociate from the catalytically inactive pre-formed gefitinib-, AG1478- and erlotinib-induced EGFR dimers (grey solid and dotted lines for EGFR homo- and EGFR-HER2 heterodimers, respectively). IM data show that the dissociation of EGF from EGFR dimers is slower compared to monomeric EGFR. The TKI induction of dimers reduces the overall dissociation rate, thus increasing the apparent affinity. At room temperature and $37^{\circ} \mathrm{C}$, but not at $7^{\circ} \mathrm{C}$, the two EGF dependently formed homo- and heterodimers (black solid and dotted line) are rapidly internalized. EGF is only visibly excreted from A431 and $\mathrm{U} 343$ cells at $37^{\circ} \mathrm{C}$, indicating that full degradation requires a physiological temperature and that EGFR-HER2 heterodimers (black, dotted line) are degraded to a lesser extent compared to EGFR homodimers (black, solid line). Gefitinib-, AG1478- and erlotinib-induced dimers have a perturbed internalization and may also be degraded more slowly. Lapatinib strengthens the monomeric EGFR form, thus reducing the overall number of dimers, which may have caused the lower degree of internalization (Fig. 2, grey solid line) as EGFR monomers are not likely to internalize. We strongly believe that a subset of internalized, TKI-induced or naturally formed EGFR dimers may recycle to the surface, although this remains to be investigated. Recycling is likely to be less in cells with a high degradation and excretion level.

As discussed previously, measurements of interactions in cells are often conducted at non-physiological temperatures to reduce the effect of other cell activities. This is a convenient strategy and often necessary to isolate information on the interaction itself in a measurable manner. In this study, the problem is approached from a different perspective by utilizing cell processes such as internalization and excretion present during measurement, thereby providing additional information on the receptor system. The variation of temperature is a means to turn on and off some of these processes, rendering it possible to separate and investigate these processes a few at a time. The authors understand that the method and approach used in this paper are novel and different from the conventional ones in the field. Findings reported in this paper are consistent with findings of previous studies, strengthening the credibility of our results. Following the labeled ligand in real time is a convenient and information-rich approach to estimate the combined rates of internalization, degradation and excretion to identify the rate-limiting step with minimal labor time. The use of complementing time-resolved assay set-ups to deconvolute complex receptor biology processes, as exemplified for the EGF-EGFR system in this paper, is a powerful strategy to advance the field of molecular oncology.

In summary, this study provides additional information on the intricate interplay of EGF and EGFR through the real-time analysis of the cell association of ${ }^{125} \mathrm{I}-\mathrm{EGF}$. Analyses in the presence of the TKIs rendered the comparison of the impact of the EGF-EGFR interaction on the kinetics, as well as the evaluation of the way the cell processes subsequently leading to ${ }^{125} \mathrm{I}$ excretion were altered, possible. By applying IM on the continuous data additional information was obtained on the manner in which the TKIs alter EGFR dimer levels. This study also confirms some of the previous findings described in the literature, providing additional information to create a broad understanding of effects on the EGFR system induced by the TKIs gefitinib, lapatinib, AG1478 and erlotinib, using completely different assays.

\section{Acknowledgements}

The authors would like to thank Rick Filonzi for encouraging scientific discussions on the need for comparing protein interactions at different temperatures.

\section{References}

1. Scaltriti M and Baselga J: The epidermal growth factor receptor pathway: a model for targeted therapy. Clin Cancer Res 12: 5268-5272, 2006

2. Yarden $Y$ and Sliwkowski MX: Untangling the ErbB signalling network. Nat Rev Mol Cell Biol 2: 127-137, 2001.

3. Blume-Jensen P and Hunter T: Oncogenic kinase signalling. Nature 411: 355-365, 2001.

4. Ferguson KM, Berger MB, Mendrola JM, Cho HS, Leahy DJ and Lemmon MA: EGF activates its receptor by removing interactions that autoinhibit ectodomain dimerization. Mol Cell 11: 507-517, 2003.

5. Garrett TP, McKern NM, Lou M, et al: Crystal structure of a truncated epidermal growth factor receptor extracellular domain bound to transforming growth factor alpha. Cell 110: 763-773, 2002.

6. Ogiso H, Ishitani R, Nureki O, et al: Crystal structure of the complex of human epidermal growth factor and receptor extracellular domains. Cell 110: 775-787, 2002.

7. Zhang X, Gureasko J, Shen K, Cole PA and Kuriyan J: An allosteric mechanism for activation of the kinase domain of epidermal growth factor receptor. Cell 125: 1137-1149, 2006.

8. Liu P, Sudhaharan T, Koh RM, et al: Investigation of the dimerization of proteins from the epidermal growth factor receptor family by single wavelength fluorescence cross-correlation spectroscopy. Biophys J 93: 684-698, 2007.

9. Tao RH and Maruyama IN: All EGF(ErbB) receptors have preformed homo- and heterodimeric structures in living cells. J Cell Sci 121: 3207-3217, 2008.

10. Björkelund H, Gedda L, Barta P, Malmqvist M and Andersson K: Gefitinib induces epidermal growth factor receptor dimers which alters the interaction characteristics with ${ }^{125}$ I-EGF. PLoS One 6: e24739,

11. Bollig-Fischer A, Dziubinski M, Boyer A, Haddad R, Giroux CN and Ethier SP: HER-2 signaling, acquisition of growth factor independence, and regulation of biological networks associated with cell transformation. Cancer Res 70: 7862-7873, 2010.

12. Worthylake R, Opresko LK and Wiley HS: ErbB-2 amplification inhibits down-regulation and induces constitutive activation of both ErbB-2 and epidermal growth factor receptors. J Biol Chem 274: 8865-8874, 1999.

13. Tzahar E, Waterman H, Chen X, et al: A hierarchical network of interreceptor interactions determines signal transduction by Neu differentiation factor/neuregulin and epidermal growth factor. Mol Cell Biol 16: 5276-5287, 1996. 
14. Björkelund $\mathrm{H}$, Gedda L and Andersson K: Comparing the epidermal growth factor interaction with four different cell lines: intriguing effects imply strong dependency of cellular context. PLoS One 6: e16536, 2011.

15. Lax I, Bellot F, Howk R, Ullrich A, Givol D and Schlessinger J: Functional analysis of the ligand binding site of EGF-receptor utilizing chimeric chicken/human receptor molecules. EMBO J 8: 421-427, 1989

16. Ullrich A and Schlessinger J: Signal transduction by receptors with tyrosine kinase activity. Cell 61: 203-212, 1990.

17. Ozcan F, Klein P, Lemmon MA, Lax I and Schlessinger J: On the nature of low- and high-affinity EGF receptors on living cells Proc Natl Acad Sci USA 103: 5735-5740, 2006.

18. Baselga J: Targeting tyrosine kinases in cancer: the second wave. Science 312: 1175-1178, 2006.

19. Ciardiello F: Epidermal growth factor receptor tyrosine kinase inhibitors as anticancer agents. Drugs 60 (Suppl 1): 25-32; Discussion 41-22, 2000

20. Baselga J and Averbuch SD: ZD1839 ('Iressa') as an anticancer agent. Drugs 60 (Suppl 1): 33-40; Discussion 41-42, 2000.

21. Pollack VA, Savage DM, Baker DA, et al: Inhibition of epidermal growth factor receptor-associated tyrosine phosphorylation in human carcinomas with CP-358,774: dynamics of receptor inhibition in situ and antitumor effects in athymic mice. J Pharmacol Exp Ther 291: 739-748, 1999.

22. Xia W, Liu LH, Ho P and Spector NL: Truncated ErbB2 receptor (p95ErbB2) is regulated by heregulin through heterodimer formation with ErbB3 yet remains sensitive to the dual EGFR/ ErbB2 kinase inhibitor GW572016. Oncogene 23: 646-653, 2004

23. Kris MG, Natale RB, Herbst RS, et al: Efficacy of gefitinib, an inhibitor of the epidermal growth factor receptor tyrosine kinase in symptomatic patients with non-small cell lung cancer: a randomized trial. JAMA 290: 2149-2158, 2003.

24. Petrelli F, Borgonovo K, Cabiddu M and Barni S: Efficacy of EGFR tyrosine kinase inhibitors in patients with EGFR-mutated non-small cell lung cancer: a meta-analysis of 13 randomized trials. Clin Lung Cancer 13: 107-114, 2012.

25. Jamal-Hanjani M and Spicer J: Epidermal growth factor receptor tyrosine kinase inhibitors in the treatment of epidermal growth factor receptor-mutant non-small cell lung cancer metastatic to the brain. Clin Cancer Res 18: 938-944, 2012.

26. Kwak EL, Sordella R, Bell DW, et al: Irreversible inhibitors of the EGF receptor may circumvent acquired resistance to gefitinib. Proc Natl Acad Sci USA 102: 7665-7670, 2005.

27. Nishimura Y, Bereczky B and Ono M: The EGFR inhibitor gefitinib suppresses ligand-stimulated endocytosis of EGFR via the early/late endocytic pathway in non-small cell lung cancer cell lines. Histochem Cell Biol 127: 541-553, 2007.

28. Bublil EM and Yarden Y: The EGF receptor family: spearheading a merger of signaling and therapeutics. Curr Opin Cell Biol 19: 124-134, 2007.

29. Lichtner RB, Menrad A, Sommer A, Klar U and Schneider MR Signaling-inactive epidermal growth factor receptor/ligand complexes in intact carcinoma cells by quinazoline tyrosine kinase inhibitors. Cancer Res 61: 5790-5795, 2001

30. Arteaga CL, Ramsey TT, Shaw ver LK and Guyer CA: Unliganded epidermal growth factor receptor dimerization induced by direct interaction of quinazolines with the ATP binding site. J Biol Chem 272: 23247-23254, 1997

31. Stamos J, Sliwkowski MX and Eigenbrot C: Structure of the epidermal growth factor receptor kinase domain alone and in complex with a 4-anilinoquinazoline inhibitor. J Biol Chem 277: 46265-46272, 2002.
32. Wood ER, Truesdale AT, McDonald OB, et al: A unique structure for epidermal growth factor receptor bound to GW572016 (Lapatinib): relationships among protein conformation, inhibitor off-rate, and receptor activity in tumor cells. Cancer Res 64 6652-6659, 2004.

33. Barros FF, Powe DG, Ellis IO and Green AR: Understanding the HER family in breast cancer: interaction with ligands, dimerization and treatments. Histopathology 56: 560-572, 2010.

34. Wells A: EGF receptor. Int J Biochem Cell Biol 31: 637-643, 1999.

35. Haslekas C, Breen K, Pedersen KW, Johannessen LE, Stang E and Madshus IH: The inhibitory effect of ErbB2 on epidermal growth factor-induced formation of clathrin-coated pits correlates with retention of epidermal growth factor receptor-ErbB2 oligomeric complexes at the plasma membrane. Mol Biol Cell 16: 5832-5842, 2005.

36. Hommelgaard AM,Lerdrup M and van Deurs B: Association with membrane protrusions makes ErbB2 an internalization-resistant receptor. Mol Biol Cell 15: 1557-1567, 2004.

37. Lenferink AE, Pinkas-Kramarski R, van de Poll ML, et al: Differential endocytic routing of homo- and hetero-dimeric ErbB tyrosine kinases confers signaling superiority to receptor heterodimers. EMBO J 17: 3385-3397, 1998.

38. Westermark B, Magnusson A and Heldin CH: Effect of epidermal growth factor on membrane motility and cell locomotion in cultures of human clonal glioma cells. J Neurosci Res 8: 491-507, 1982.

39. Carlsson J: Potential for clinical radionuclide-based imaging and therapy of common cancers expressing EGFR-family receptors. Tumour Biol 33: 653-659, 2012.

40. Björkelund $\mathrm{H}$, Gedda L and Andersson K: Avoiding false negative results in specificity analysis of protein-protein interactions. $\mathrm{J}$ Mol Recognit 24: 81-89, 2010.

41. Sundberg AL, Almqvist Y, Orlova A, et al: Combined effect of gefitinib ('Iressa', ZD1839) and targeted radiotherapy with 211At-EGF. Eur J Nucl Med Mol Imaging 30: 1348-1356, 2003.

42. Nordberg E, Friedman M, Gostring L, et al: Cellular studies of binding, internalization and retention of a radiolabeled EGFR-binding affibody molecule. Nucl Med Biol 34: 609-618, 2007.

43. Bjorke $\mathrm{H}$ and Andersson $\mathrm{K}$ : Automated, high-resolution cellular retention and uptake studies in vitro. Appl Radiat Isot 64: 901-905, 2006.

44. Hunter WM and Greenwood FC: Preparation of iodine-131 labelled human growth hormone of high specific activity. Nature 194: 495-496, 1962.

45. Bjorke $\mathrm{H}$ and Andersson $\mathrm{K}$ : Measuring the affinity of a radioligand with its receptor using a rotating cell dish with in situ reference area. Appl Radiat Isot 64: 32-37, 2006.

46. Svitel J, Balbo A, Mariuzza RA, Gonzales NR and Schuck P: Combined affinity and rate constant distributions of ligand populations from experimental surface binding kinetics and equilibria. Biophys J 84: 4062-4077, 2003.

47. Barta P, Malmberg J, Melicharova L, et al: Protein interactions with HER-family receptors can have different characteristics depending on the hosting cell line. Int J Oncol 40: 1677-1682, 2012.

48. Huber W: A new strategy for improved secondary screening and lead optimization using high-resolution SPR characterization of compound-target interactions. J Mol Recognit 18: 273-281, 2005. 